\title{
Design and Synthesis of a Structurally Constrained Aminoglycoside
}

\author{
Dale Kling, Dusan Hesek, Qicun Shi, and Shahriar Mobashery* \\ Department of Chemistry and Biochemistry, University of Notre Dame, IN 46556 USA \\ mobashery@nd.edu
}

\section{Supporting Information}

Table of Contents.

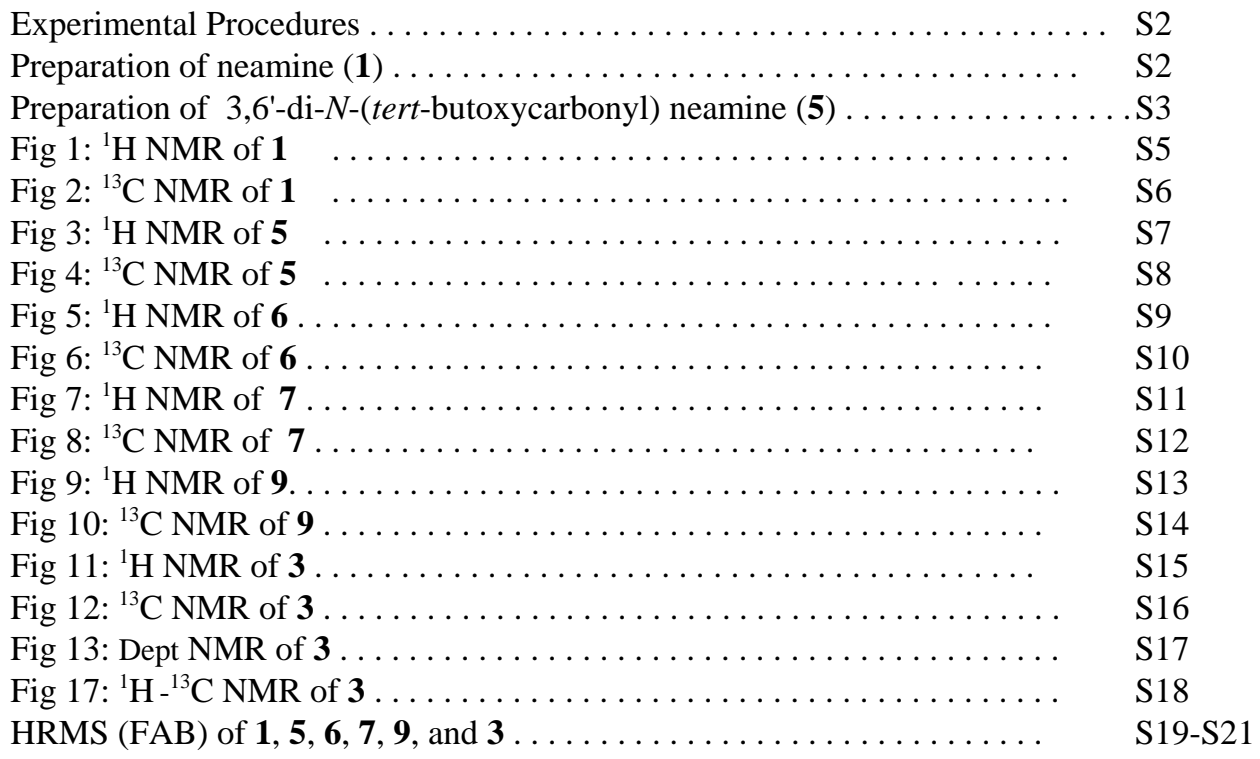




\section{EXPERIMENTAL PROCEDURES}

Various 1D and 2D homo- and hetero-nuclear NMR techniques including ${ }^{1} \mathrm{H}$-relay, ${ }^{1} \mathrm{H}-{ }^{1} \mathrm{H}$

COSY, ${ }^{1} \mathrm{H}-{ }^{13} \mathrm{C}$ HETCOR, and ${ }^{1} \mathrm{H}-{ }^{13} \mathrm{C}$ gHMBC were utilized to elucidate the structures of the constrained aminoglycoside 3, and the corresponding intermediates 5, 6, 7, and 9.

MS electrospray of the purified derivatives were carried out on LC/MS instrument consisting of chromatography module, photodiode array detector 2996, and mass spectrometer, using a Pro C18 YMC reverse phase column $(3 \times 50 \mathrm{~mm})$. The mobile phase was $10 \mathrm{mM}$ ammonium acetate in HPLC grade water (A), and HPLC grade acetonitrile (B). A gradient was formed from $5 \%$ to $80 \%$ of $\mathrm{B}$ in 10 minutes, and the flow rate of the LC was $0.7 \mathrm{~mL} / \mathrm{min}$. The MS electrospray source operated at capillary voltage $3.5 \mathrm{kV}$ and a desolvation temperature $300{ }^{\circ} \mathrm{C}$.

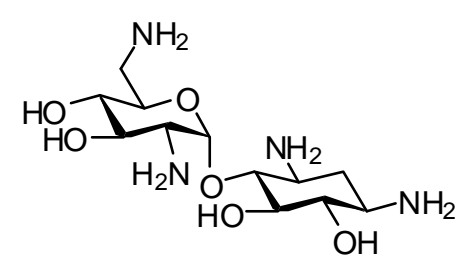

1

Preparation of Neamine ${ }^{1,2}$ (1). A solution of $20 \mathrm{~g}$ (22 mmol) of neomycin B sulfate (4) in $1 \mathrm{~L}$ of anhydrous methanol was refluxed for 6 hours under a nitrogen atmosphere with $215 \mathrm{ml}$ of $1.8 \mathrm{~N}$ methanolic hydrogen chloride. The flask was then cooled to room temperature before placement in a refrigerator overnight. Refrigeration caused the precipitation of $5.6 \mathrm{~g}$ of an amorphous white solid (1 $\mathrm{HCl})$, which was isolated via vacuum filtration. The mother liquor from the precipitation was concentrated to $300 \mathrm{ml}$ and diluted with $200 \mathrm{ml}$ anhydrous ether to afford another $1.8 \mathrm{~g}$ of tan colored solid $\mathbf{1}$. HCl. Free base of neamine $\mathbf{1}$ was achieved by taking $5.6 \mathrm{~g}$ of the solid hydrochloride $\mathbf{1}$ up in $6.3 \mathrm{ml}$ of concentrated ammonium hydroxide, and diluting it with $350 \mathrm{ml}$ of methanol. Ammonia gas was then passed through the solution until crystallization began. This solution was then refrigerated 
overnight. The following day crystals were collected via vacuum filtration on a Buchner funnel, washing three times with $20 \mathrm{ml}$ portions of cold anhydrous methanol and drying in vacuo. This resulted in the isolation of $5.1 \mathrm{~g}(16 \mathrm{mmol})$ of neamine free base; a 70\% overall yield from neomycin B sulfate (4). ${ }^{1} \mathrm{H}\left(\mathrm{D}_{2} \mathrm{O}, 500 \mathrm{MHz}\right) \delta 5.12(\mathrm{~d}, J=4 \mathrm{~Hz}, 1 \mathrm{H}$, H-1'), 3.60 (m, 1H, H-5'), 3.39 (m, 2H, H-3', H-4), 2.97-3.2 (m, 3H, H-4', H-5, H-6), 2.84 (dd, $\omega=16 \mathrm{~Hz}, J=13.5 \mathrm{~Hz}, 2.5 \mathrm{~Hz}, 1 \mathrm{H}, \mathrm{H}-6^{\prime}$ ), 2.52-2.73 (m, 4H, H-1, H-2', H-3, H-6'), $1.81\left(\mathrm{ddd}, \omega=21 \mathrm{~Hz}\right.$, assigned as a 1:2:1:1:2:1 6 line $\mathrm{m}, J_{2 \mathrm{eq}-2 \mathrm{ax}}=13 \mathrm{~Hz}, J_{2 \mathrm{eq}-1}=J_{2 \mathrm{eq}-3}=$ $4 \mathrm{~Hz}, 1 \mathrm{H}, \mathrm{H}-2 \mathrm{eq}), 1.04\left(\mathrm{ddd}, \omega=37 \mathrm{~Hz}\right.$, assigned as a 1:3:3:1 4 line $\mathrm{m}, J_{2 \mathrm{ax}-2 \mathrm{eq}}=13 \mathrm{~Hz}$, $\left.J_{2 \mathrm{ax}-1}=J_{2 \mathrm{ax}-3}=12 \mathrm{~Hz}, 1 \mathrm{H}, \mathrm{H}-2 \mathrm{ax}\right) ;{ }^{13} \mathrm{C}$ NMR $\left(\mathrm{D}_{2} \mathrm{O}, 125 \mathrm{MHz}\right) \delta 100.6\left(\mathrm{C}-1^{\prime}\right), 86.7$ (C-5), 77.4 (C-6), 76.1 (C-4), 73.6 (C-3'), 72.5 (C-5'), 71.5 (C-4'), 55.3 (C-2'), 50.5 (C-1), 49.4 (C-3), $41.6(\mathrm{C}-2), 35.5\left(\mathrm{C}-6{ }^{\prime}\right)$. MS (FAB+) $\mathrm{m} / z 323[\mathrm{M}+\mathrm{H}]^{+}$calcd for $\mathrm{C}_{12} \mathrm{H}_{26} \mathrm{~N}_{4} \mathrm{O}_{6}$ is 322.3; M.p. $=255-256^{\circ} \mathrm{C}$, Lit. $256^{\circ} \mathrm{C}$.

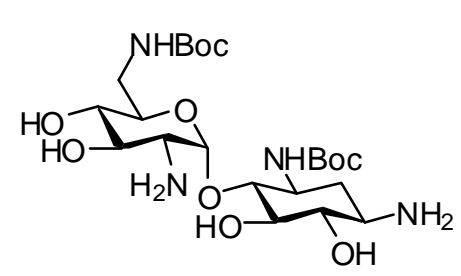

5
3,6'-Di- $N$-(tert-butoxycarbonyl)-neamine ${ }^{3} \quad$ (5). Neamine free base $(\mathbf{1}, 5 \mathrm{~g}, 15 \mathrm{mmol})$ was dissolved in a minimal amount of water $(10 \mathrm{ml})$ followed by the addition of $1 \mathrm{~L}$ DMSO. Copper (II) acetate monohydrate (12.4 g, $60 \mathrm{mmol})$

was then added and the blue solution was allowed to stir overnight before adding di-tertbutyldicarbonate $(7.43 \mathrm{~g}, 34 \mathrm{mmol})$. The solution was stirred for an additional $24 \mathrm{~h}$, after which time TLC analysis $\left(\mathrm{CHCl}_{3} / \mathrm{MeOH} /\right.$ concentrated ammonia, 2:1:0.25) revealed the presence of 3 spots, one at $\mathrm{R}_{f}=0.7$, one at $\mathrm{R}_{f}=0.5$ and one at $\mathrm{R}_{f}=0.1$ corresponding to the tricarbamoyl species, dicarbamoyl species and monocarbamoyl species, respectively. DMSO was removed by adding $800 \mathrm{ml}$ ethyl ether, mixing vigorously for 15 minutes and 
decanting the upper layer. This process was repeated multiple times using fresh $500 \mathrm{ml}$ portions of ether until a dark oily residue remained. The residue was then dissolved in $600 \mathrm{ml}$ in $\mathrm{MeOH} / \mathrm{H}_{2} \mathrm{O}$ (4:1) and copper was precipitated by saturating the solution with hydrogen sulfide gas. Solid was filtered off through a layer of celite and the filtrate was evaporated to obtain a brown residue, which was taken up in a minimal amount of $\mathrm{MeOH}$ and chromatographed using the same solvent system as in the initial TLC. A mass of 3.94 $\mathrm{g}$ of title compound 5 was obtained providing an overall yield of $49 \% .{ }^{1} \mathrm{H} \mathrm{NMR}\left(\mathrm{CD}_{3} \mathrm{OD}\right.$, $500 \mathrm{MHz}) \delta 5.18\left(\mathrm{~d}, J=4 \mathrm{~Hz}, 1 \mathrm{H}, \mathrm{H}-1^{\prime}\right), 3.66$ (unresolved multiplet, 1H, H-5'), 3.45 (overlapping multiplet, 3H, H-3, H-3', H-4 or H-5), 3.38 (t, $J=9 \mathrm{~Hz}, 1 \mathrm{H}, \mathrm{H}-4$ or H-5), 3.30 (broad, 2H, H-6') 3.13 (t, $J=9.5 \mathrm{~Hz}, 1 \mathrm{H}, \mathrm{H}-4$ '), 3.06 (t, $J=9.5 \mathrm{~Hz}, 1 \mathrm{H}, \mathrm{H}-6), 2.55$ (overlapping multiplet, $\left.2 \mathrm{H}, \mathrm{H}-1, \mathrm{H}-2^{\prime}\right) 1.98(\mathrm{ddd}, \omega=21 \mathrm{~Hz}$, assigned as a 1:2:1:1:2:1 6 line $\left.\mathrm{m}, J_{2 \mathrm{eq}-2 \mathrm{ax}}=13 \mathrm{~Hz}, J_{2 \mathrm{eq}-1}=J_{2 \mathrm{eq}-3}=4 \mathrm{~Hz}, 1 \mathrm{H}, \mathrm{H}-2 \mathrm{eq}\right), 1.41(\mathrm{~s}, 18 \mathrm{H}, \mathrm{OC}(C H 3) 3), 1.29$ (ddd, $\omega=37$, assigned as a 1:3:3:1 4 line $\mathrm{m}, J_{2 \mathrm{ax}-2 \mathrm{eq}}=13 \mathrm{~Hz}, J_{2 \mathrm{ax}-1}=J_{2 \mathrm{ax}-3}=12 \mathrm{~Hz}, 1 \mathrm{H}$, H-2ax); ${ }^{13} \mathrm{C}$ NMR $\left(\mathrm{CD}_{3} \mathrm{OD}, 125 \mathrm{MHz}\right) \delta 101.9$ (C-1'), 84.1 (C-4), 78.6 (C-6), 77.6 (C-5), $74.7\left(\mathrm{C}-3^{\prime}\right), 72.6$ and 72.4 (C-4' and C-5'), 57.1 (C-2'), 52.4 (C-1), 50.9 (C-3), 42.1 (C-6'),

$36.3(\mathrm{C}-2), 29.0(\mathrm{OC}(\mathrm{CH} 3) 3)$. HRMS $\left(\mathrm{FAB}^{+}\right)$for $\mathrm{C}_{22} \mathrm{H}_{42} \mathrm{~N}_{4} \mathrm{O}_{10}[\mathrm{M}+\mathrm{H}]^{+}$: calcd 523.2979, found 523.2968.

\section{References}

1. Dutcher, J.; Donin, M. J. Am. Chem. Soc. 1952, 74, 3420-3422.

2. Ford, J.; Bergy, M.; Brooks, A.; Garrett, E.; Alberti, J.; Dyer, J.; Carter, H. J. Am. Chem. Soc. 1955, 77, 5311-5314.

3. Grapsas, I.; Massova, I.; Mobashery, S. Tetrahedron 1998, 54, 7705-7720. 


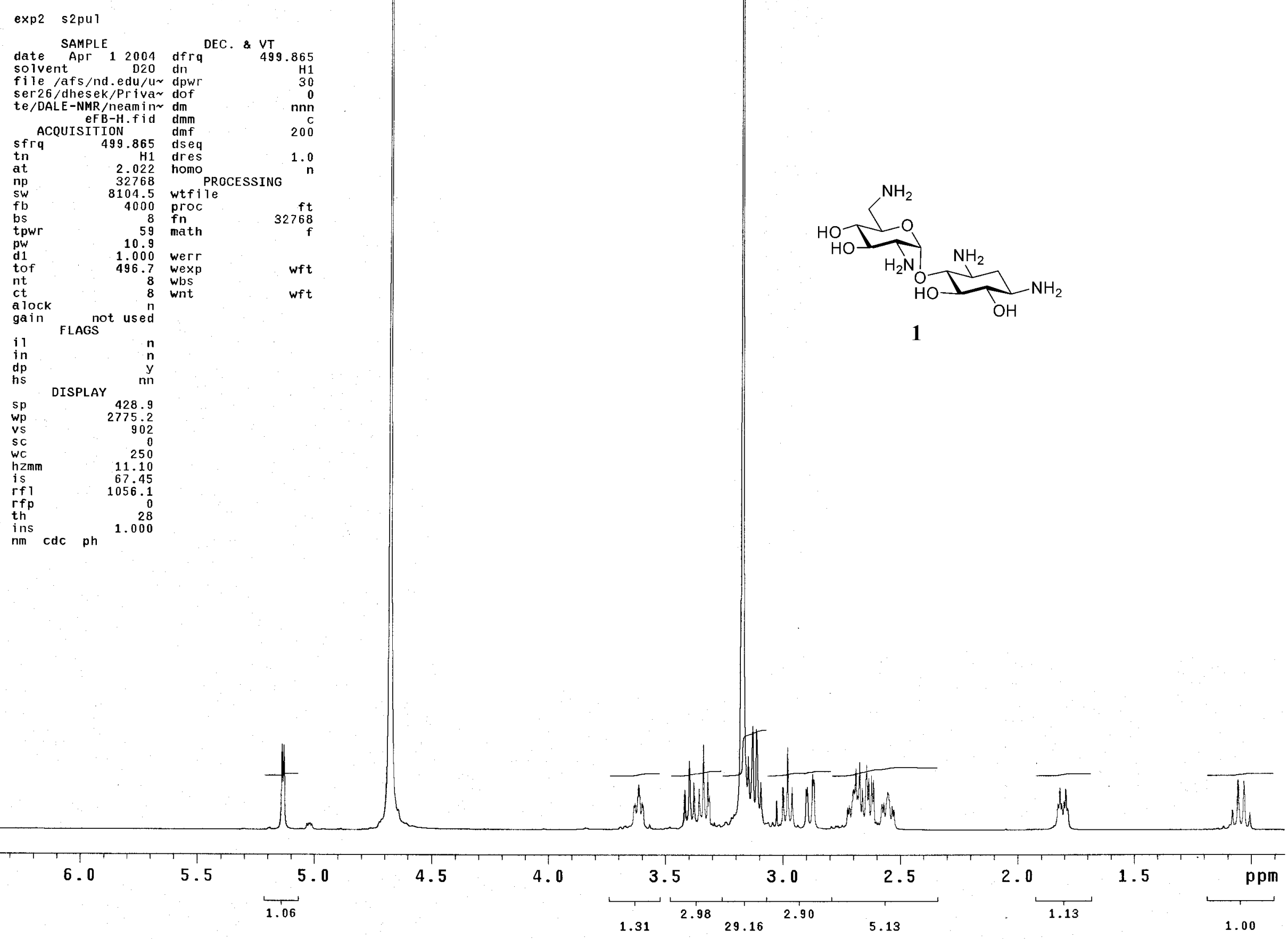


Fig 2: $13 \mathrm{C}$ NMR of 1
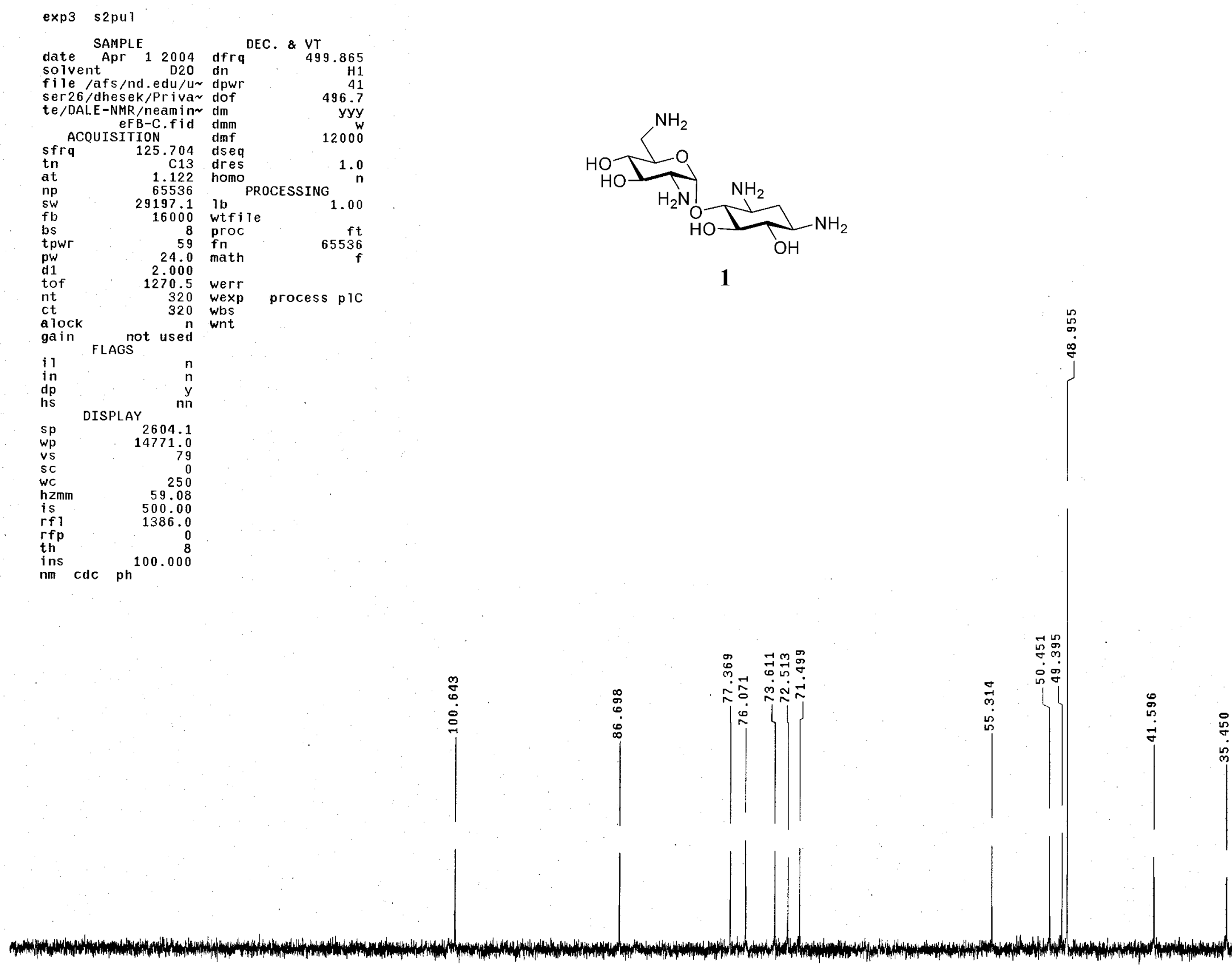


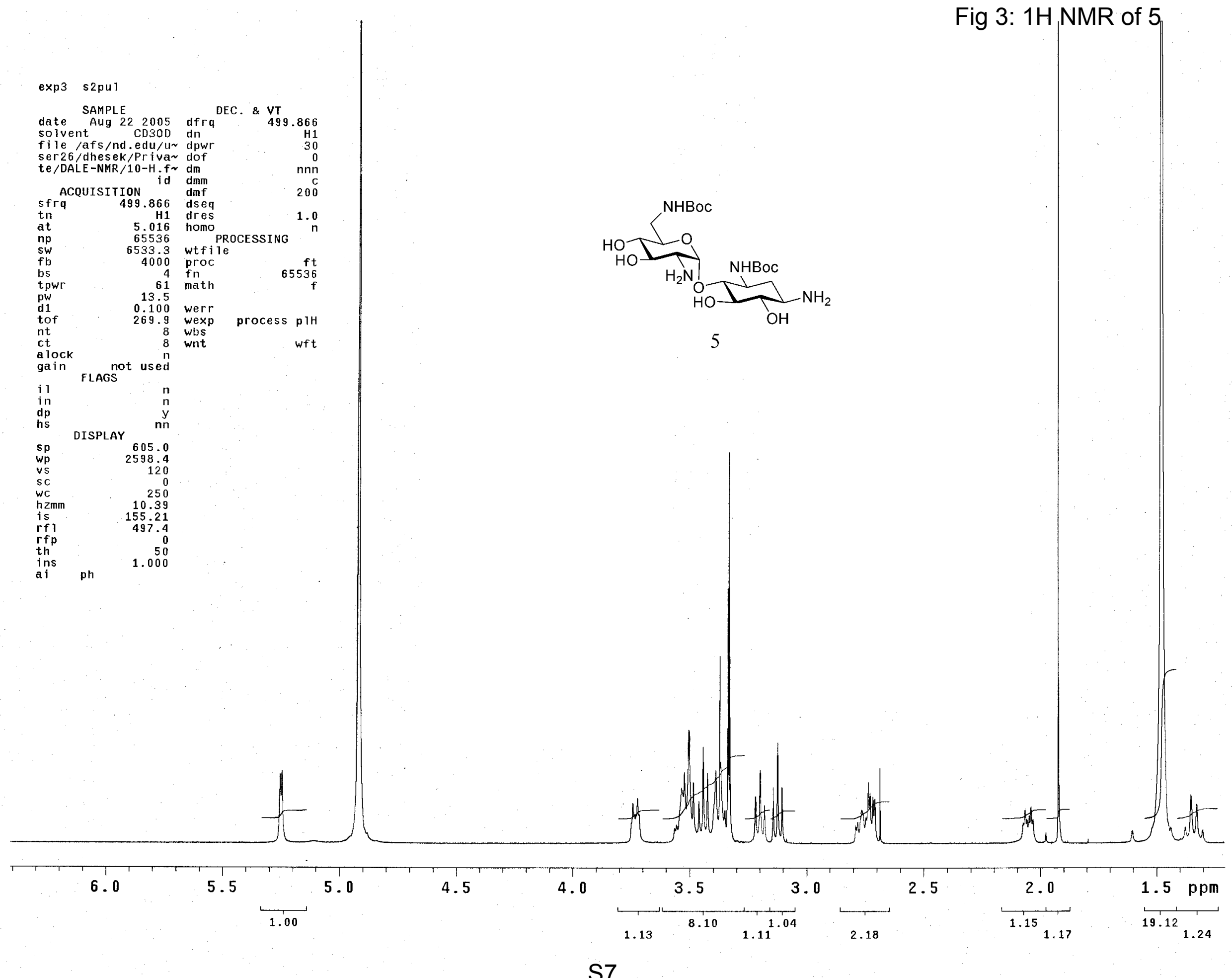


Fig 4: 13C NMR of 5

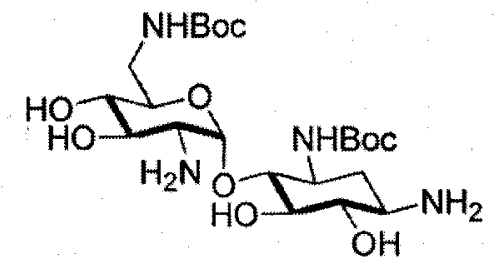

5

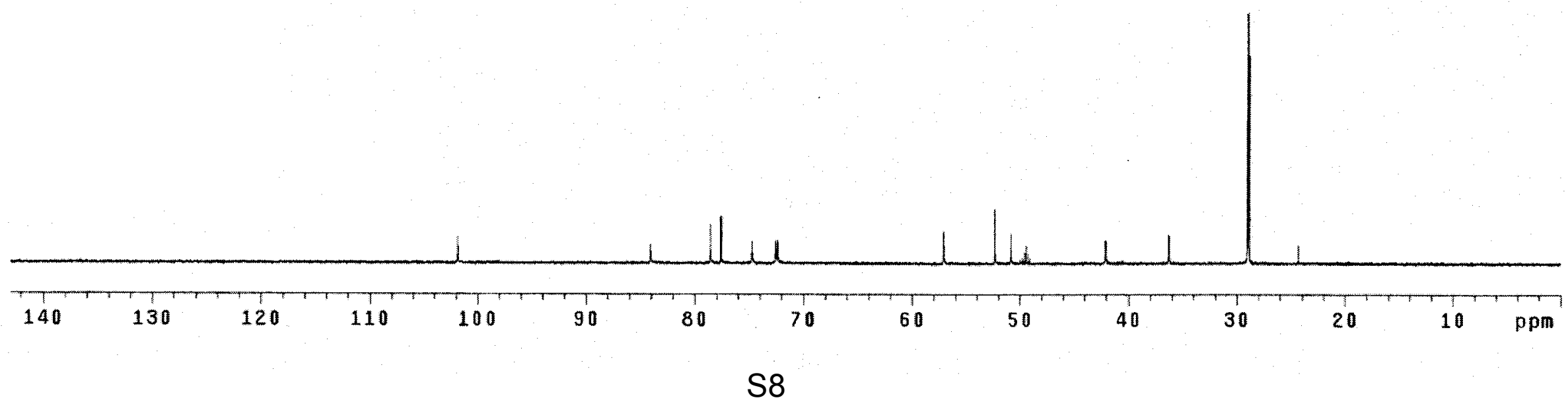


$\exp 3 \quad s 2 p u 1$

date $\begin{gathered}\text { SAMPLE } \\ \text { Aug } 22\end{gathered} 2005$ dfrq DEC. \& VT

solvent CD30D dn

file /afs/nd.edu/ur dpw

te/DALE-NMR/11-H.f $\sim$ dm

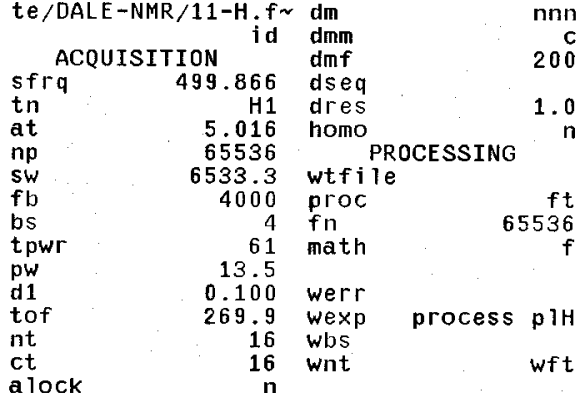

866
$H 1$
30
0
0

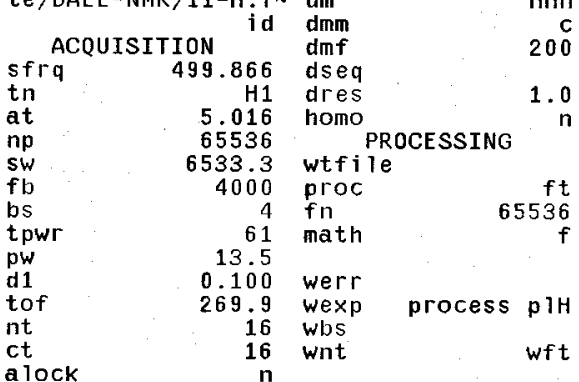

alock not used
gain

$\begin{array}{lr}\text { il } & n \\ \text { in } & n \\ \text { dp } & y \\ \text { hs } & \text { nn }\end{array}$

DISPLAY

$$
\underset{\mathrm{nn}}{\mathrm{n}}
$$

$\begin{array}{rr}5 p & 584.3 \\ \text { wp } & 4137.9\end{array}$

$\begin{array}{ll}\text { VS } & 171 \\ \text { SC } & 0\end{array}$

$\begin{array}{rr}\text { WC } & 250 \\ \text { hzmm } & 16.55\end{array}$

$\begin{array}{lr}\text { is } & 192.06 \\ \mathrm{rf} 7 & 497.4\end{array}$

$\begin{array}{rr}r f p & 0 \\ t h & 50\end{array}$

ins $\quad 10.000$

ai ph

$\therefore$

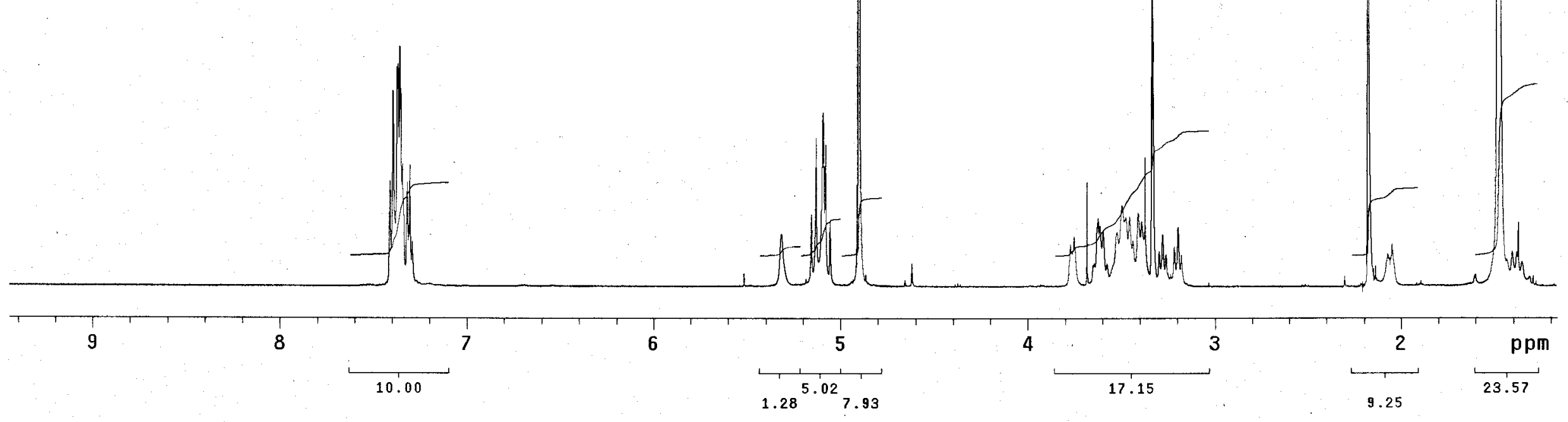


Fig 6: $13 \mathrm{C}$ NMR of 6
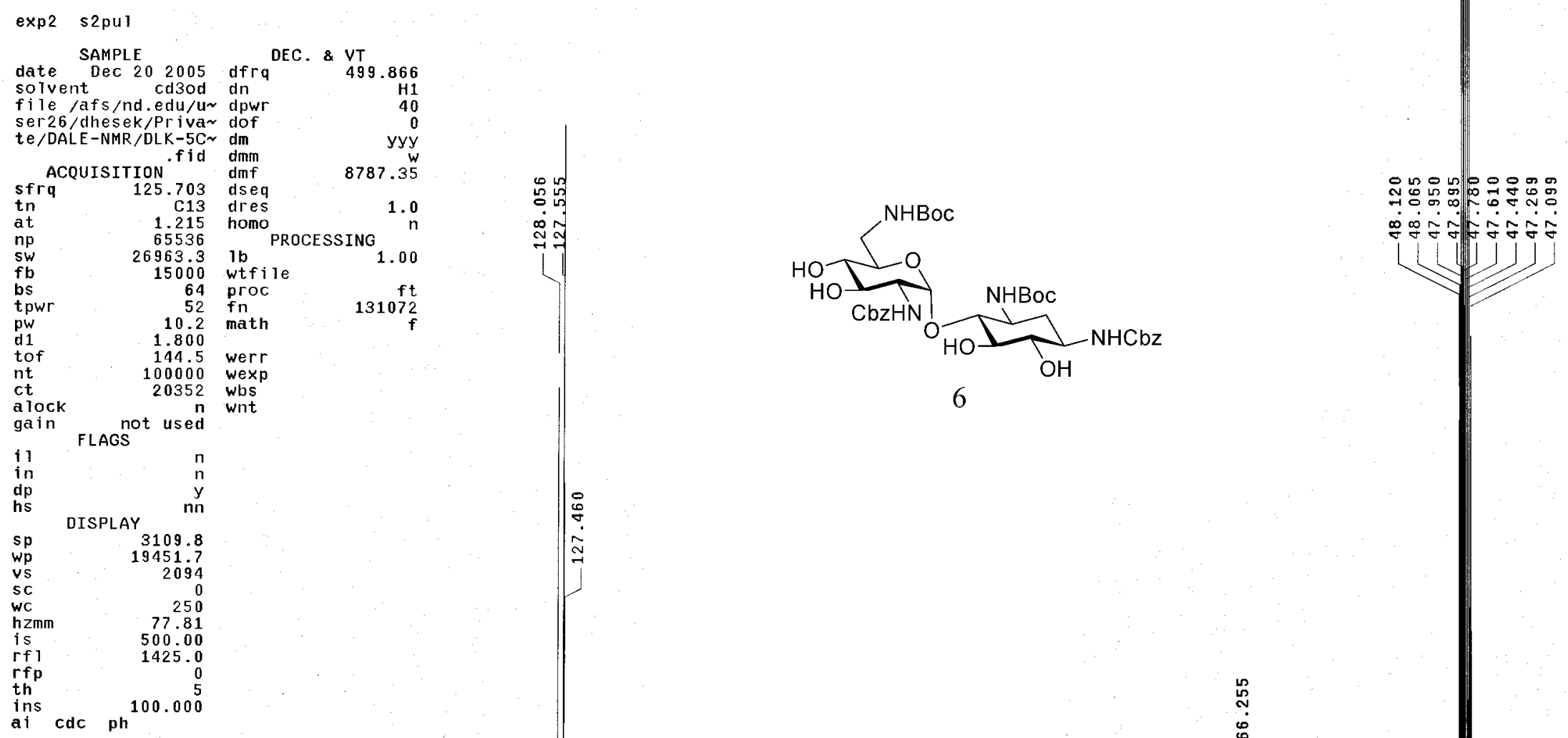
Fig 7: $1 \mathrm{H}$ NMR of 7

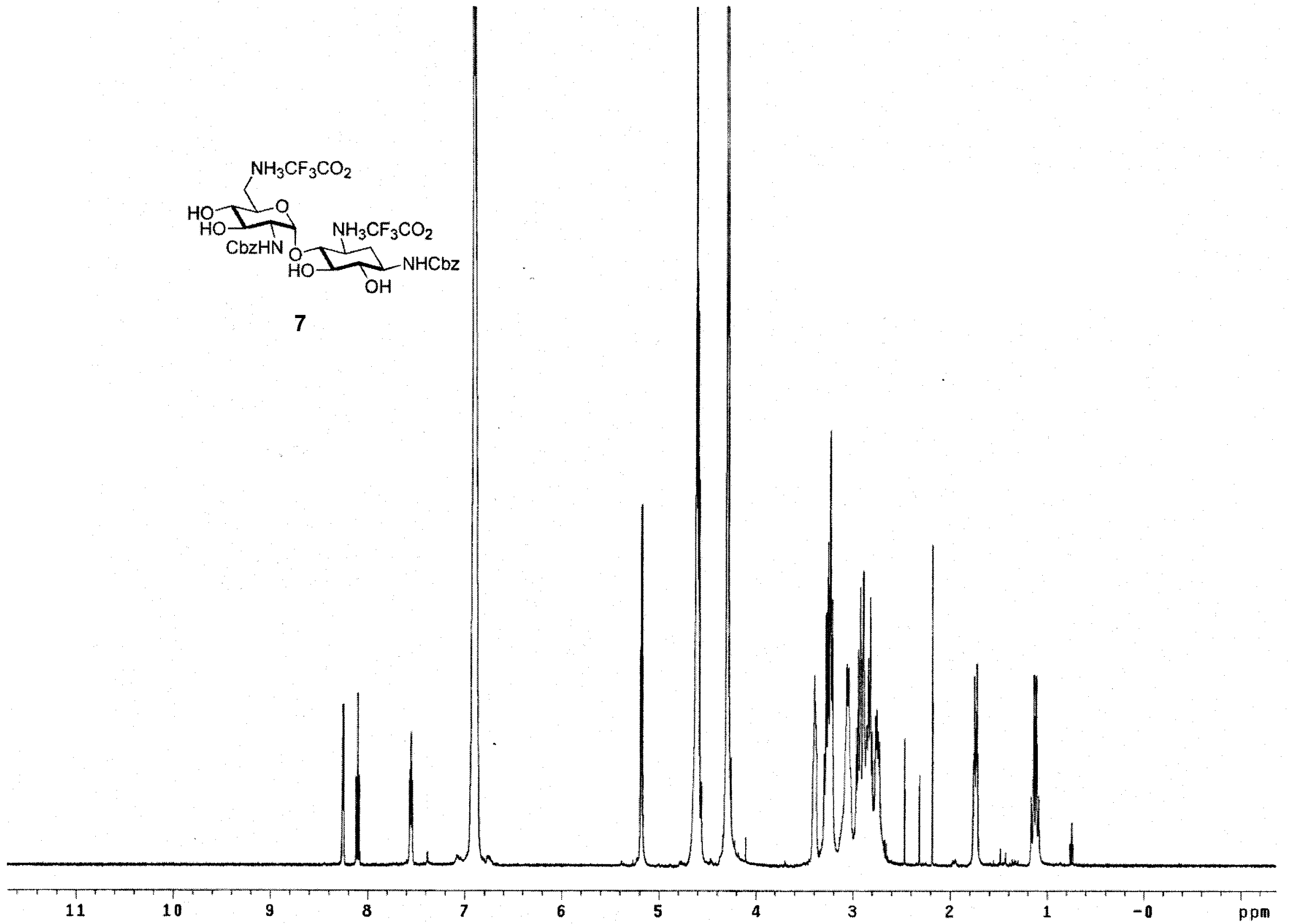


exp3 s2pul

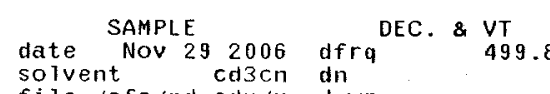

file /afs/nd.edu/ur dpwr

ser26/dhesek/Privar dof

te/DALE-NMR/12-C.f $\sim$ dm

ACQUisition id dimn

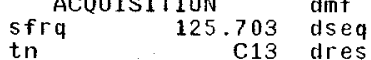

$\begin{array}{lll}\text { tn } & \text { c13 dres } \\ \text { at } & 1.215 & \text { homo }\end{array}$

np $\quad 265536$

$\begin{array}{rrr}\text { fb } & 26963.3 & \mathrm{dfrq} \\ \mathrm{bs} & 15000 & \mathrm{dn} 2\end{array}$

$\begin{array}{lrl}\text { bs } & 4 & \text { dpwr2 } \\ \text { tpwr } & 52 & \text { dof }\end{array}$

tpw

dpw
tof

tof

nt
ct
a lock

10.2
1.800

144.5 dmf

1648 dseq2

gain not used

$\begin{array}{lll}\text { in } & \text { FLAGS } & \text { dfrq } \\ \text { in } & & \text { dn } 3 \\ \text { di } & \text { dpwr }\end{array}$

n dpwr

hS DISPLAY

$\begin{array}{cl}y & \text { dof } 3 \\ \text { nn } & d m 3\end{array}$

$\begin{array}{rrr}\mathrm{sp} & \text { DISPLAY } & \mathrm{dmm} \\ \mathrm{wp} & 3561.2 & \mathrm{dmf} \\ \mathrm{vs} & 18743.0 & \mathrm{dsea}\end{array}$

$\begin{array}{rrr}\text { wp } & 18743.0 & \text { dseq3 } \\ \text { vs } & 998 & \text { dres }\end{array}$

wC

hzmm 74.97

250 PROCESSING

$\begin{aligned} 74.97 & \text { lb } \\ 500.00 & \text { wtfi }\end{aligned}$

1467.6 proc

$\begin{array}{rrr}\mathrm{rfp} & 174.7 \mathrm{fn} \\ \mathrm{th} & 4 \mathrm{math}\end{array}$

ins

ins cdc ph

100.000

werr
wexp

$\substack{w e x \\ \text { wbs } \\ \text { wht }}$
the

Fig 8: $13 \mathrm{C}$ NMR of 7

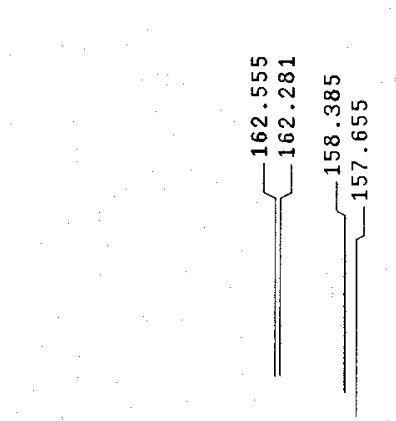

$\mathrm{ft}$
131072
$\mathrm{f}$

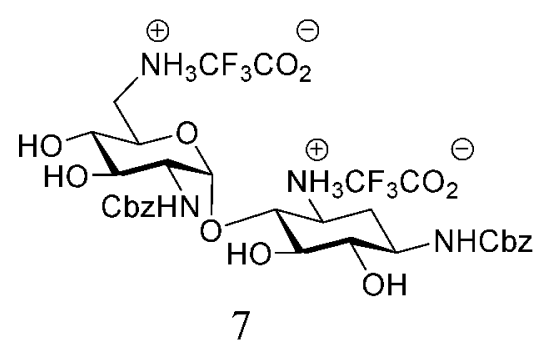

急

$\infty$
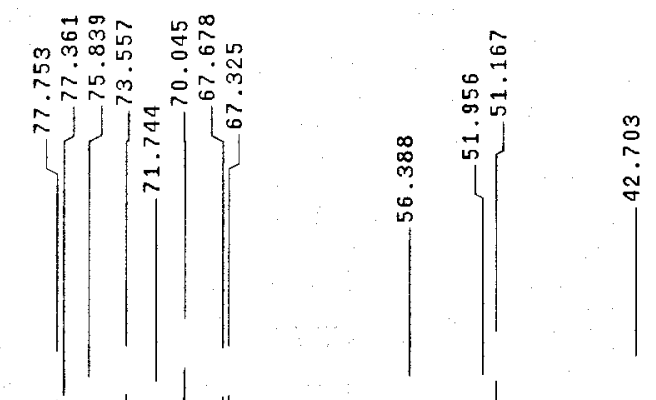


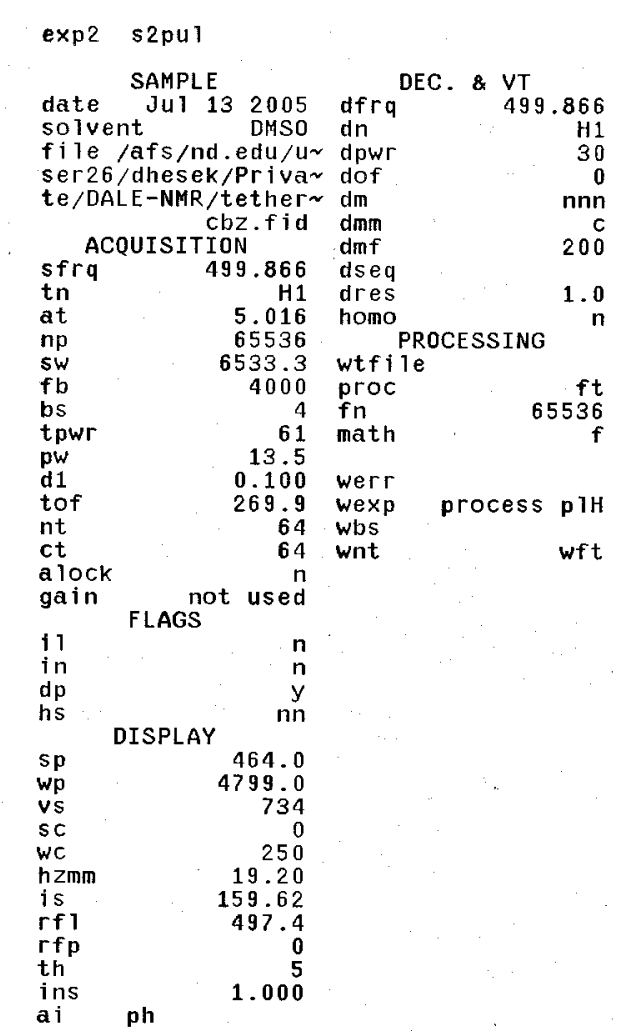

Fig 9: $1 \mathrm{H}$ NMR of 9

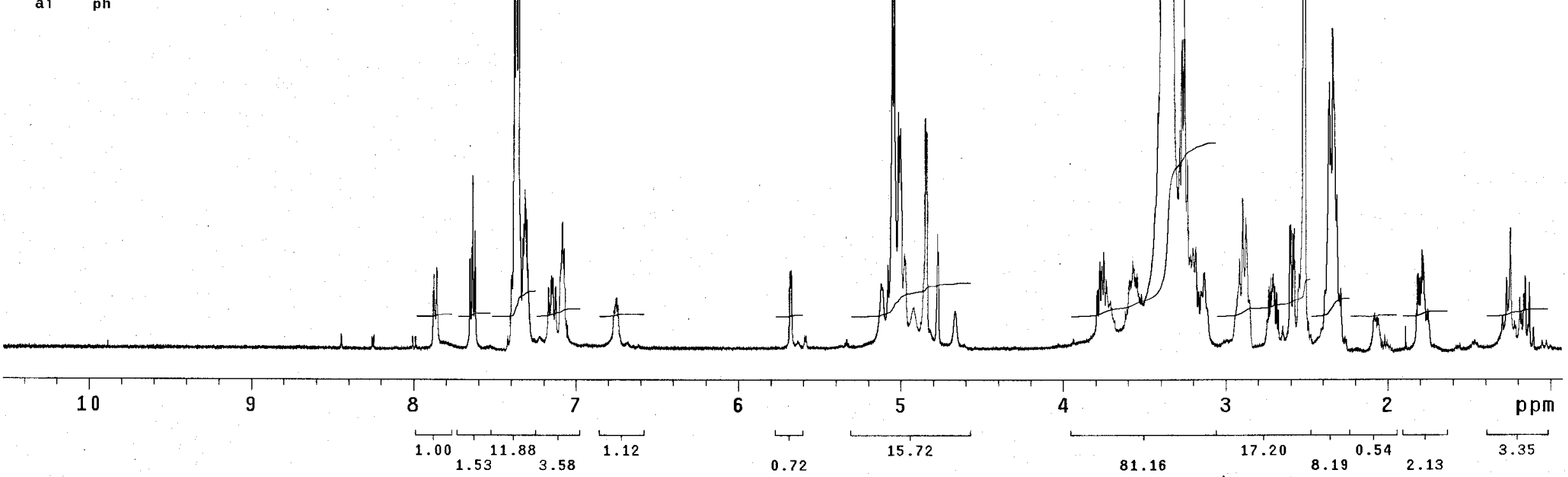


Fig 10: 13C NMR of 9

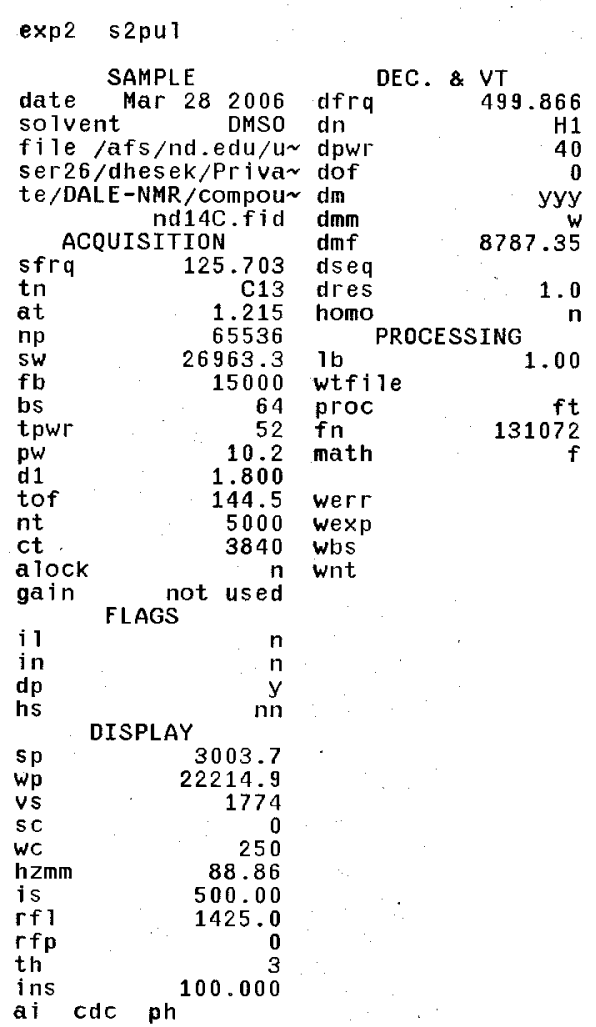




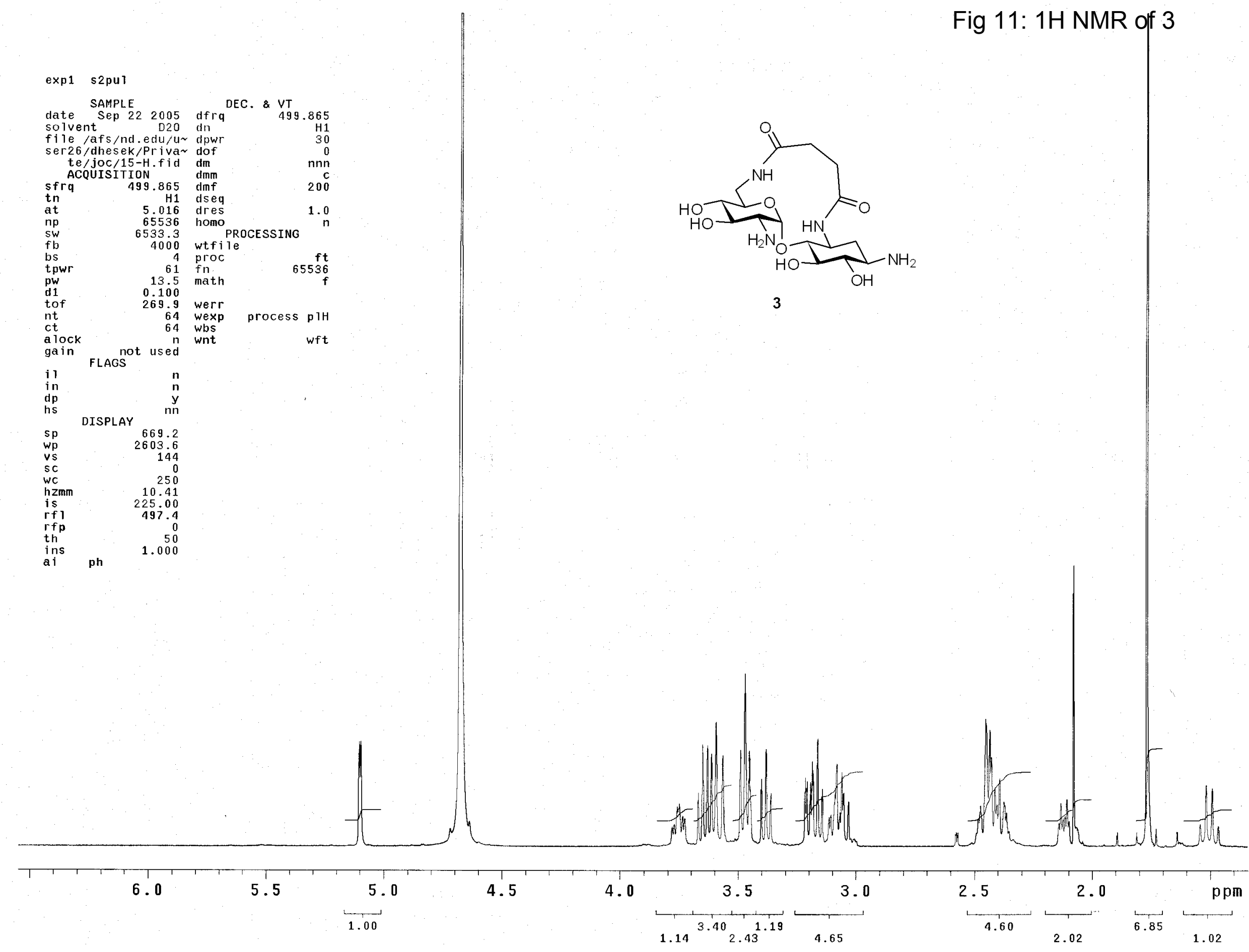


Fig 12: 13C NMR of 3

15

exp2 s2pul

SAMPLE 2005 DEC. \& VT

date Sep 222005 dfrq 20 dn 49.865

$\begin{array}{ll}\text { solvent } & \text { D20 dn } \\ \text { file /afs/nd.edu/ur dpwr } & 40\end{array}$

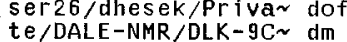

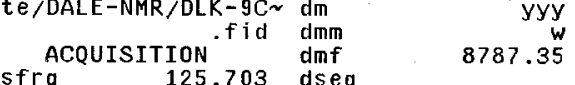

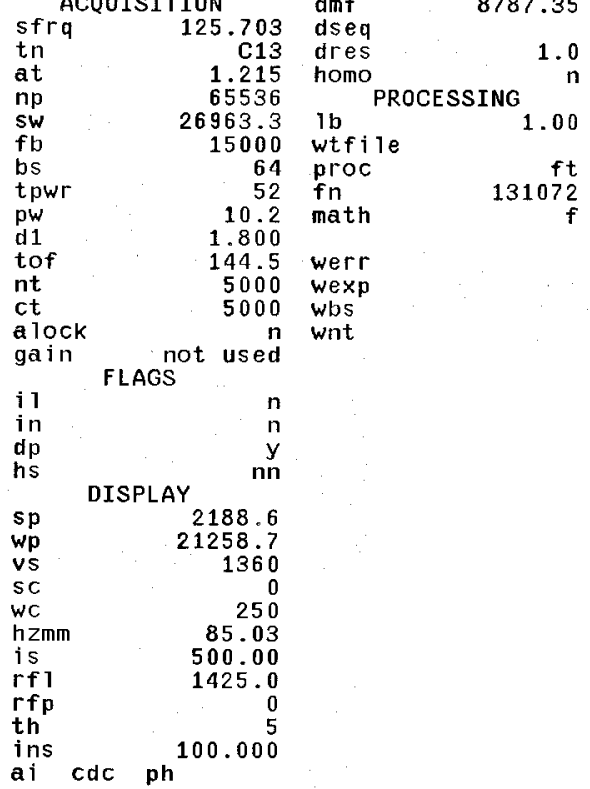
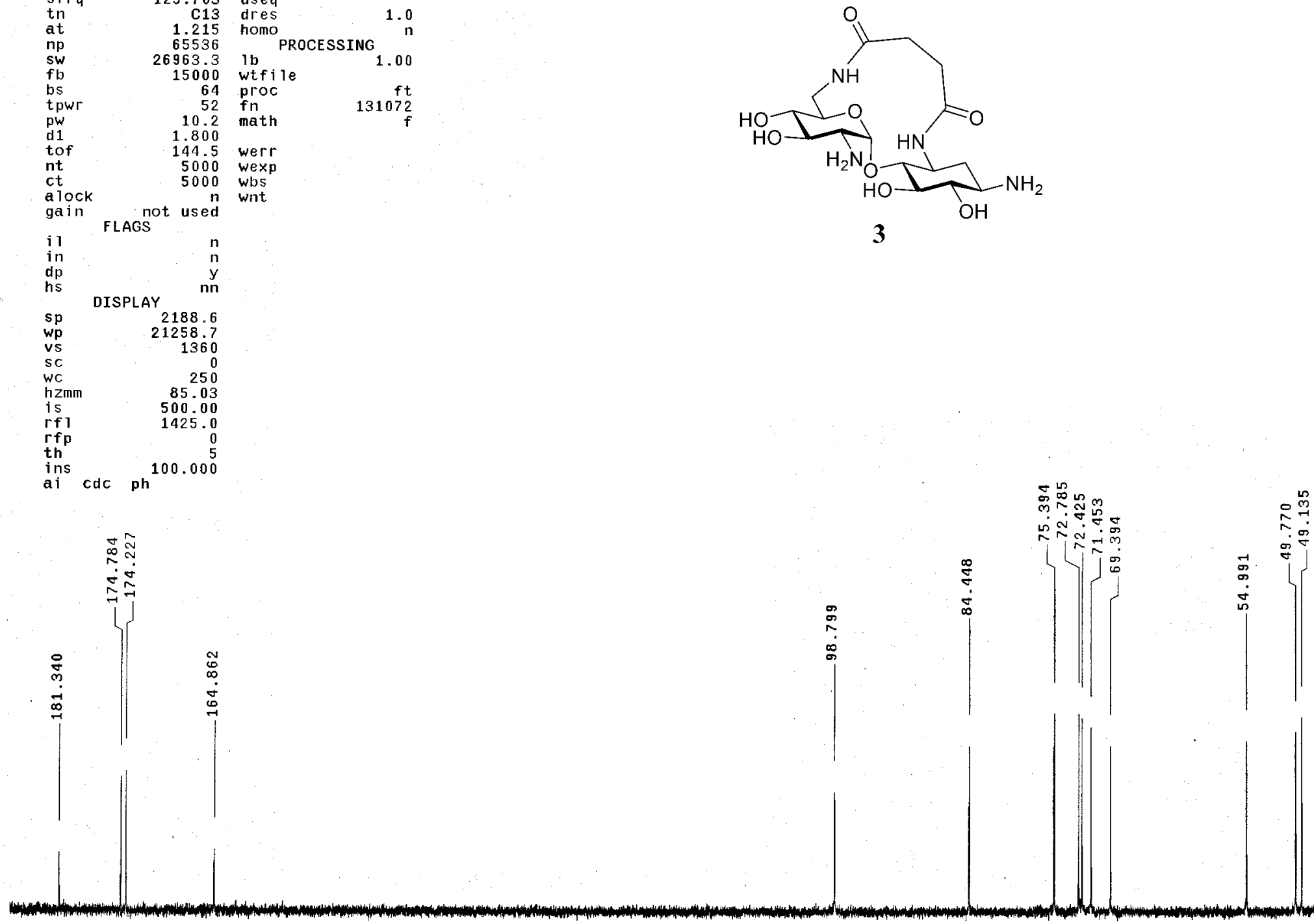

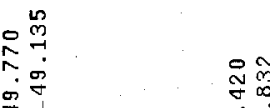
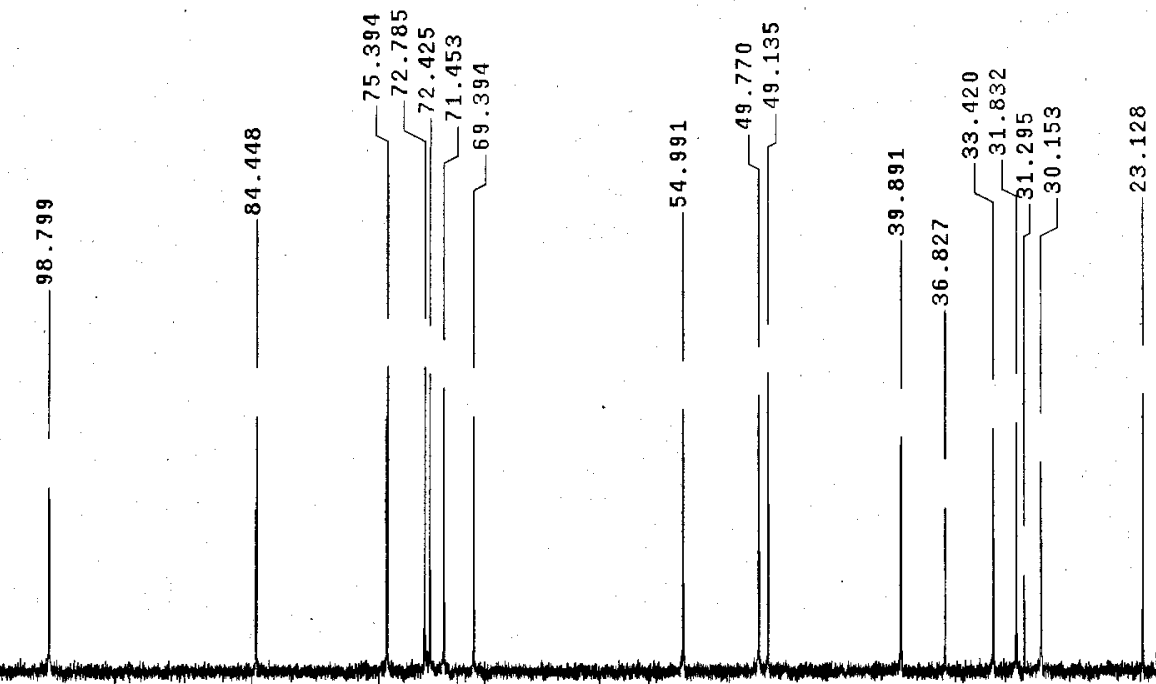

180

160

140

120

100

80

60

40

ppm 


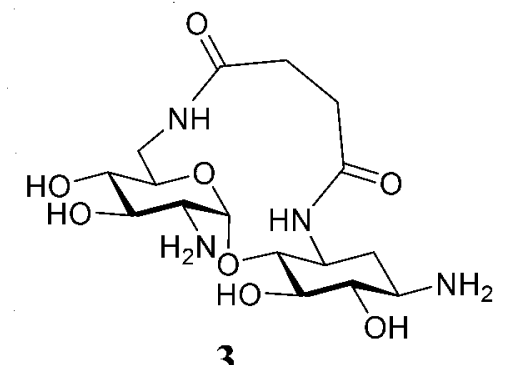

$\mathrm{CH} 2$ carbons
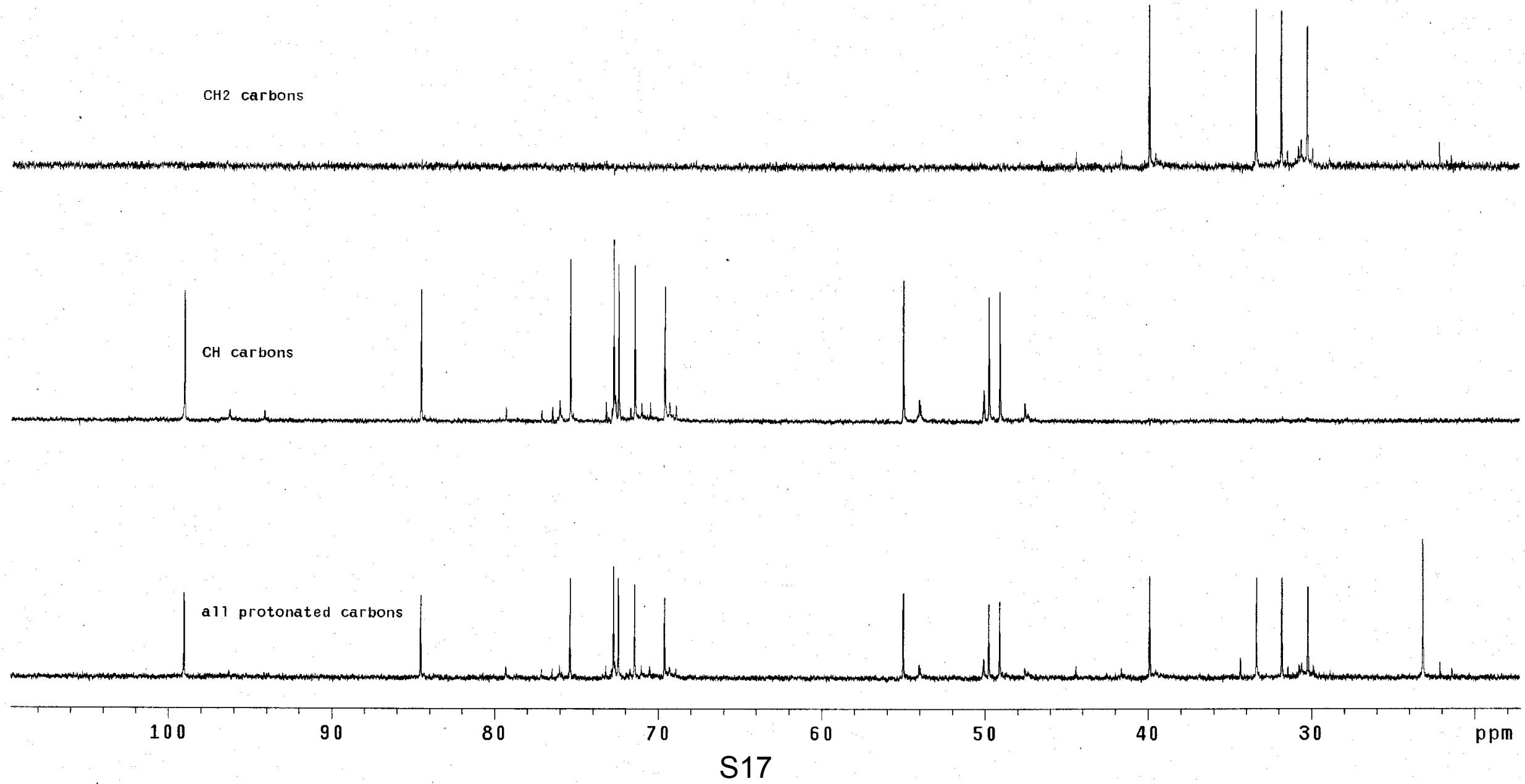
Fig 17: $1 \mathrm{H}-13 \mathrm{C}$ NMR of 3

Pulse Sequence: hetcor

Solvent: D20

Ambient temperature

User: 1-14-87

I leva-500

Relax. delay $1.500 \mathrm{sec}$

Acq. time $0.111 \mathrm{sec}$

2D Width $6533.3 \mathrm{~Hz}$

132 repetitions

256 increments

OBSERVE C13, $125.6905122 \mathrm{MHZ}$

Power $40 \mathrm{~dB}$. 4052

on during acquisit

WALTZ-16 modulated

DATA PROCESSING

Li ine broadening $1.0 \mathrm{~Hz}$

Line broadening $0.3 \mathrm{~Hz}$

FT size $4096 \times 1024$
Total time $15 \mathrm{hr}, 33 \mathrm{~min}, 25 \mathrm{sec}$

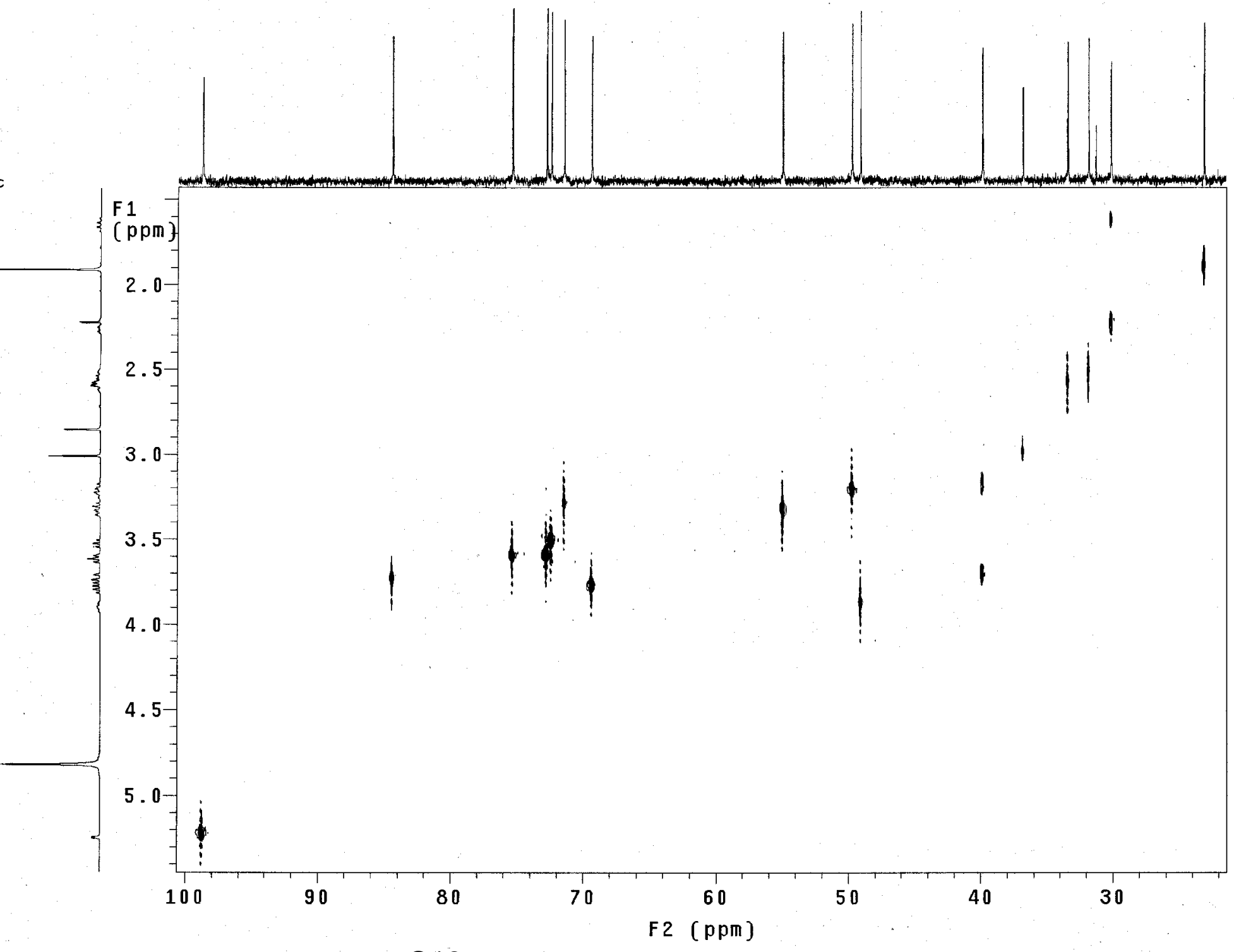

$\mathrm{S} 18$ 
[ Theoretical Ion Distribution ]

Molecular Formula : $\mathrm{C} 12 \mathrm{H} 27$ N4 06

(m/z 323.1931, MW 323.3696, U.S. 1.5)

Base Peak : 323.1931, Averaged MW : $323.3705(\mathrm{a}), \quad 323.3711(\mathrm{w})$

$\mathrm{m} / \mathrm{z}$ INT.

323.1931100 .0000

324.195915 .4498

$325.19802 .3193 *$

$326.2004 \quad 0.2355$

$327.2026 \quad 0.0210$

$328.2049 \quad 0.0016$

$329.2072 \quad 0.0001$

[ Elemental Composition ]

Data : J6.2484hr-001

Date : $30-$ Nov-2006 10:42

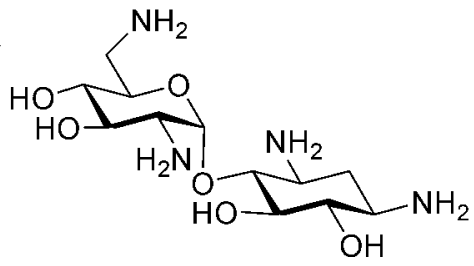

Sample: DSH-11

Note : - in PEG standard

Inlet : Reserv.

RT : $0.71 \mathrm{~min}$

Elements : C 40/0, H 49/0, O 7/0, N 5/0

Ion Mode : FAB+

Scan\# : $(1,11)$

Mass Tolerance : $3 \mathrm{mmu}$

Unsaturation (U.S.) : $-0.5-5.0$

Observed $\mathrm{m} / \mathrm{z}$ Int $\frac{\%}{\operatorname{Err}}[\mathrm{ppm} / \mathrm{mmu}]$ U.S. Composition

$\begin{array}{lllllllllllll}323.1938 & 100.0 & -1.7 / & -0.6 & 1.0 & \mathrm{C} & 14 & \mathrm{H} & 29 & \mathrm{O} & 7 & \mathrm{~N} & \\ & & +2.4 / & +0.8 & 1.5 & \mathrm{C} & 12 & \mathrm{H} & 27 & \mathrm{O} & 6 & \mathrm{~N} & 4\end{array}$

[ Theoretical Ion Distribution ]

Molecular Formula : C22 H43 N4 O1O

$(\mathrm{m} / \mathrm{z} \quad 523.2979$, MW 523.6042, U.S. 3.5)

Base Peak : 523.2979, Averaged MW : 523.6057 (a), 523.6064 (w)

$\mathrm{m} / \mathrm{z}$ INT.

523.2979100 .0000

524.301026 .9645

$525.3034 \quad 5.5013$

$526.3059 \quad 0.8301$

$527.3083 \quad 0.1053$

$528.3107 \cdot 0.0115$

$529.3131 \quad 0.0011$

[ Elemental Composition ]

Data : J6.2483hr-002

Sample: DSH-14

Note : - in PEG standard

Inlet : Reserv.

$\mathrm{RT}: 0.68 \mathrm{~min}$

Elements : C $40 / 0, \mathrm{H} 49 / 0, \mathrm{O} 11 / 0, \mathrm{~N} 5 / 0$

Date : $30-$ Nov-2006 10:37

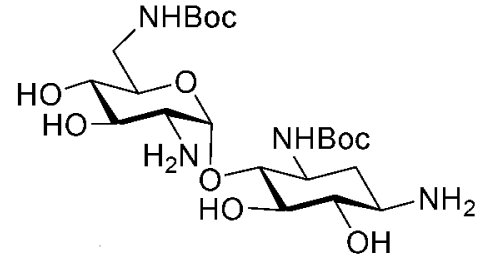

5

Mass Tolerance : $3 \mathrm{mmu}$

Unsaturation (U.S.) : $-0.5-12.0$

Observed $\mathrm{m} / \mathrm{z}$ Int $\frac{\circ}{0} \operatorname{Err}[\mathrm{ppm} / \mathrm{mmu}]$ U.S. Composition

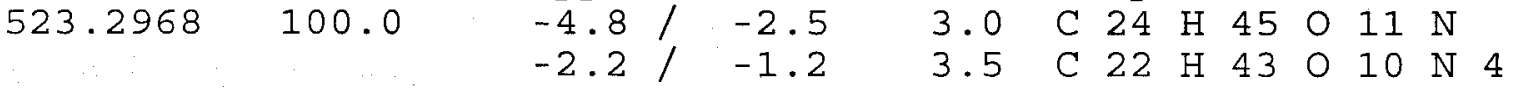


[ Theoretical Ion Distribution ]

\section{HRMS (FAB) of 6 and 7}

Molecular Formula : C38 H54 N4 $014 \mathrm{Na}$

$(\mathrm{m} / \mathrm{z}, 813.3534$, MW 813.8549, U.S. 13.5)

Base Peak : 813.3534 , Averaged MW : $813.8570(\mathrm{a}), \quad 813.8577(\mathrm{w})$

$\mathrm{m} / \mathrm{z} \quad$ INT.

813.3534100 .0000

$814.3566 \quad 45.0777$

$815.359412 .7288 * * * * * * *$

$816.3620 \quad 2.6853 * *$

$817.3646 \quad 0.4635$

$818.3672 \quad 0.0684$

$819.3697 \quad 0.0089$

$820.3721 \quad 0.0010$

$821.3746 \quad 0.0001$

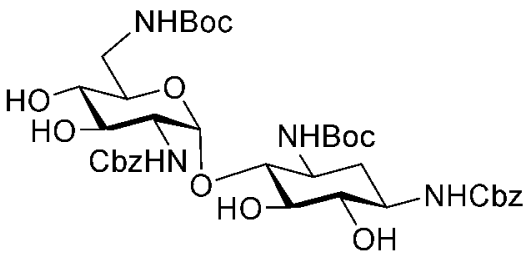

6

[ Elemental Composition ]

Data : J6.2486hr-001

Date : 30-Nov-2006 11:03

Sample: DLK-11

Note : - in PEG standard

Inlet : Reserv.

Ion Mode : FAB+

$\mathrm{RT}: 0.87 \mathrm{~min}$

Scan\# : $(1,10)$

Elements : C 40/0, H 90/0, O 15/0, N 5/0, Na 1/0

Mass Tolerance : $3 \mathrm{mmu}$

Unsaturation (U.S.) : $-0.5-15.0$

Observed $\mathrm{m} / \mathrm{z}$ Int\% Err[ppm / mmu U.S. Composition

$\begin{array}{lllllllll}813.3535 & 100.0 & -1.5 / & -1.2 & 13.0 & \mathrm{C} 40 \mathrm{H} 56 & 0 & 15 \mathrm{~N} \mathrm{Na}\end{array}$

$+0.1 /+0.1 \quad 13.5$ C 38 H $54014 \mathrm{~N} 4 \mathrm{Na}$

[ Theoretical Ion Distribution ]

Molecular Formula : C28 H39 N4 O10

$(\mathrm{m} / \mathrm{z} \quad 591.2666, \mathrm{MW} \quad 591.6385, \mathrm{U} . \mathrm{S} .11 .5)$

Base Peak : 591.2666, Averaged MW : $591.6400(\mathrm{a}), \quad 591.6407(\mathrm{w})$

$\mathrm{m} / \mathrm{z} \quad \mathrm{INT}$.

591.2666100 .0000

$592.2698 \quad 33.5779$

593.27237 .4662

$594.2749 \quad 1.2456 *$

595.27740 .1709

$596.2799 \quad 0.0201$

$597.2823 \quad 0.0021$

$598.2848 \cdot 0.0002$

[ Elemental Composition]

Data : J6.2482hr-001

Date : 30-Nov-2006 10:30

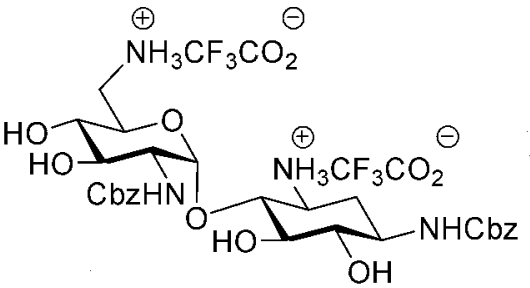

Sample: DSH-16

Note : - in PEG standard

Inlet : Reserv.

Ion Mode : $\mathrm{FAB}+$

$\mathrm{RT}: 0.29 \mathrm{~min}$

Scan\# : $(1,4)$

Elements : C 40/0, H 49/0, O 11/0, N 5/0

Mass Tolerance : $3 \mathrm{mmu}$

Unsaturation (U.S.) : $-0.5-12.0$

Observed $\mathrm{m} / \mathrm{z}$ Int\% Err [ppm / mmu U.S. Composition

$\begin{array}{lllllllllllll}591.2648 & 100.0 & -3.0 / & -1.8 & 11.5 & \mathrm{C} & 28 & \mathrm{H} & 39 & \mathrm{O} & 10 \mathrm{~N} & 4\end{array}$ 
[ Theoretical Ion Distribution ]

\section{HRMS (FAB) of 9 and 3}

Molecular Formula : C32 H41 N4 012

(m/z 673.2721, MW 673.6971, U.S. 14.5)

Base Peak : 673.2721, Averaged MW : 673.6987(a), 673.6994(w)

$\mathrm{m} / \mathrm{z}$ INT.

673.2721100 .0000

$674.2753 \quad 38.1331$

$675.27799 .4756 * \star * * *$

$676.2805 \quad 1.7656$ *

$677.2830 \quad 0.2705$

$678.2855 \quad 0.0355$

$679.2880 \quad 0.0041$

$680.2904 \quad 0.0004$

[ Elemental Composition ]

Data : J6.2485hr-002

Sample: DLK-14

Note : - in PEG standard

Inlet : Reserv.

$\mathrm{RT}: 0.57 \mathrm{~min}$

Elements : C $40 / 0, \mathrm{H} 49 / 0, \mathrm{O} 13 / 0, \mathrm{~N} 5 / 0$

Ion Mode : FAB+

Date : $30-\mathrm{Nov}-200610: 53$

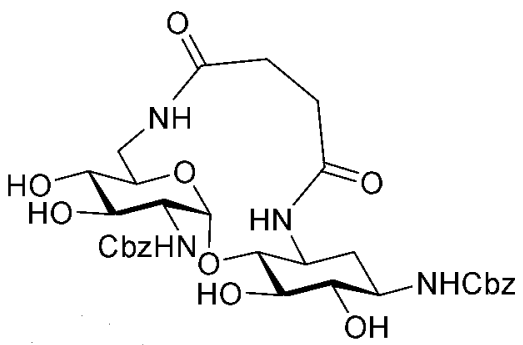

9

Mass Tolerance : $3 \mathrm{mmu}$

Unsaturation (U.S.) : $-0.5-15.0$

Observed $\mathrm{m} / \mathrm{z}$ Int: Err[ppm / mmu U.S. Composition

$\begin{array}{llllllllllll}673.2722 & 11.0 & -1.8 / & -1.2 & 14.0 & \mathrm{C} & 34 & \mathrm{H} & 43 & \mathrm{O} & 13 \mathrm{~N}\end{array}$

$+0.2 \%+0.1 \quad 14.5$ C 32 H 41012 N 4

[ Theoretical Ion Distribution ]

Molecular Formula : $\mathrm{C} 16 \mathrm{H} 29$ N4 08

$(\mathrm{m} / \mathrm{z}$ 405.1985, MW 405.4283, U.S. 4.5)

Base Peak : 405.1985, Averaged MW : 405.4292(a), 405.4299(w)

$\mathrm{m} / \mathrm{z} \quad$ INT.

405.1985100 .0000

$406.2015 \quad 20.0049$

$407.2037 \quad 3.5030 * *$

$408.2062 \quad 0.4340$

$409.2085 \quad 0.0464$

$410.2108 \quad 0.0042$

$411.2131 \quad 0.0003$

[ Elemental Composition]

Data : J6.2487hr-002

Date : 30-Nov-2006 11:15

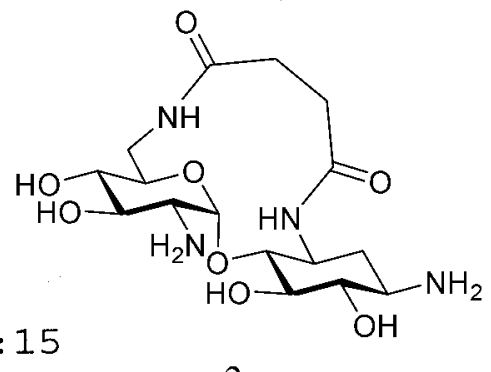

Sample: DLK-9

Note : - in PEG standard

Inlet : Reserv.

$\mathrm{RT}: 0.64 \mathrm{~min}$

Elements : C 40/0, H $90 / 0, \mathrm{O} 9 / 0, \mathrm{~N} 5 / 0$

Ion Mode : FAB+

Scan\# : $(1,10)$

Mass Tolerance : $3 \mathrm{mmu}$

Unsaturation (U.S.) : $-0.5-5.0$

Observed $\mathrm{m} / \mathrm{z}$ Int $\frac{\circ}{\circ}$ Err [ppm / mmu] $405.2003 \quad 11.7$

$$
+0.9 /+0.4
$$

U.S. Composition

$+4.2 /+1.7$

$4.0 \quad \mathrm{C} \quad 18 \mathrm{H} \quad 3109 \mathrm{~N}$

$\begin{array}{lllllllll}4.5 & \mathrm{C} & 16 & \mathrm{H} & 29 & 0 & 8 & \mathrm{~N} & 4\end{array}$ 